\title{
Parameters for selective colorimetric sensing of mercury(II) in aqueous solutions using mercaptopropionic acid-modified gold nanoparticles $\dagger$
}

\author{
Chih-Ching Huang ${ }^{a}$ and Huan-Tsung Chang*ab \\ Received (in Cambridge, UK) 23rd October 2006, Accepted 27th November 2006 \\ First published as an Advance Article on the web 11th December 2006 \\ DOI: $10.1039 / \mathrm{b} 615383 \mathrm{f}$
}

We unveil a new homogeneous assay-using mercaptopropionic acid-modified $\mathrm{Au}$ nanoparticles in the presence of 2,6pyridinedicarboxylic acid for the highly selective and sensitive detection of $\mathrm{Hg}^{2+}$ ions.

Heavy-metal ions pose severe risks for human health and the environment. ${ }^{1}$ In particular, mercury-based pollutants that arise mainly from coal-burning power plants are of great environmental concern because of the high toxicity of many $\mathrm{Hg}$ compounds. ${ }^{2}$ Chemical sensors for the detection of $\mathrm{Hg}^{2+}$ include devices based on thin films of gold, ${ }^{3}$ environmentally sensitive organic molecules, ${ }^{4}$ polymeric materials, ${ }^{5}$ and bio-composites, ${ }^{6}$ but many of these systems are of limited practical use because of such factors as poor aqueous solubility, cross-sensitivity toward other metal ions, matrix interference, and low sensitivity. Thus, the development of new and practical assays for determining $\mathrm{Hg}^{2+}$ levels in real samples remains a considerable challenge.

Metal nanoparticles are emerging as important colorimetric reporters because their extremely high visible-region extinction coefficients $\left(10^{8}-10^{10} \mathrm{M}^{-1} \mathrm{~cm}^{-1}\right)$ are often several orders of magnitude higher than those of organic dyes. ${ }^{7-12}$ In addition, differently sized and shaped $\mathrm{Au}$ nanoparticles (AuNPs) display unique optical properties. For example, 13- and 56-nm diameter AuNPs exhibit large surface plasmon extinction bands centered at 520 and $530 \mathrm{~nm}$, respectively; thus, solutions of 13- and 56-nm AuNPs are colored rose red and purple red, respectively. When 11-mercaptoundecanoic acid is self-assembled upon the 13-nm AuNP surface, its "recognition" of heavy-metal ions induces aggregation of the AuNPs. ${ }^{8}$ This process brings the AuNPs into closer proximity and the resultant coupling interactions cause a red-shifting and broadening of the surface plasmon absorption band. However, AuNP-based metal ion sensors often suffer from poor selectivity and/or low water solubility. ${ }^{8,12}$

In this study, we took advantage of the aggregation-induced color changes of 3-mercaptopropionic acid (MPA)-functionalized AuNPs (MPA-AuNPs) in aqueous solutions (in the presence of 2,6-pyridinedicarboxylic acid (PDCA)) to develop a highly selective optical sensor for $\mathrm{Hg}^{2+}$. The AuNPs $(13.3 \pm 0.5 \mathrm{~nm})$ employed as chromophores were capped with MPA through Au-S bonds. If their aggregation were to be driven by the recognition

${ }^{a}$ Department of Chemistry, National Taiwan University, Taipei, Taiwan. E-mail: changht@ntu.edu.tw; Fax:011-886-2-33661171;

Tel: 011-886-2-33661171

${ }^{b}$ Department of Natural Science Education, National Taitung

University, Taitung, Taiwan

$\dagger$ Electronic supplementary information (ESI) available: Experimental procedures on prepared compounds and Fig. S1. See DOI: 10.1039/ b615383f and binding of heavy-metal ions, the color change would allow visual sensing of the ions. Fig. 1(a) displays the UV-Vis absorption response of a $3.0 \mathrm{nM}$ suspension of MPA-AuNPs in $50 \mathrm{mM}$ Trisborate (TB) buffer ( $\mathrm{pH}$ 9.0) to the presence of $\mathrm{Hg}^{2+}$ ions. The dispersed MPA-AuNPs displayed an extinction band at $520 \mathrm{~nm}$; upon aggregation, the signal underwent a red shift with decreased extinction, while the intensity of the signal at $650 \mathrm{~nm}$ increased. The extinction coefficients at 650 and $520 \mathrm{~nm}$ are related, respectively, to the quantities of the dispersed and aggregated MPA-AuNPs. Inset a. in Fig. 1(a) indicates that the color changed from red to purple following the addition of $1.0 \mathrm{mM} \mathrm{Hg}(\mathrm{NO})_{3}$ $(100 \mu \mathrm{L})$ to the MPA-AuNP solution $(900 \mu \mathrm{L})$; the aggregated MPA-AuNPs precipitated after $1 \mathrm{~h}$. As inset b. of Fig. 1(a) suggests, by monitoring the ratio of extinction coefficients $\left(E x_{650 / 520}\right)$, the $\mathrm{Hg}^{2+}$-induced aggregation of the MPA-AuNPs reached its completion within $60 \mathrm{~min}$. Fig. 1(b) and (c) display TEM and optical dark-field scattering (DFS) images of the MPA-AuNP solutions in the absence and presence of $\mathrm{Hg}^{2+}$. Because of the low scattering intensity and faster diffusion of the 13.3-nm AuNPs, the

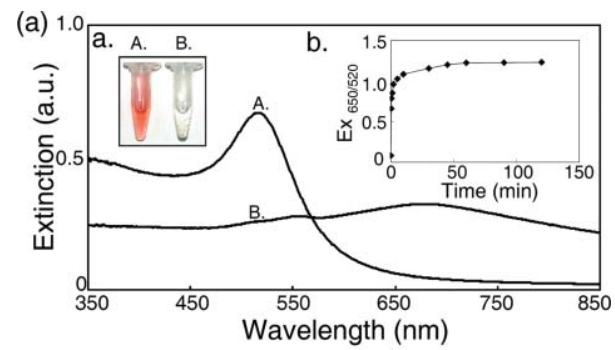

(b) $\mathrm{A}$
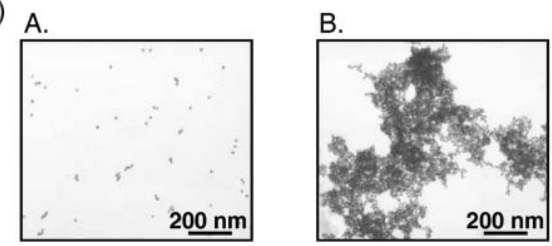

(c)
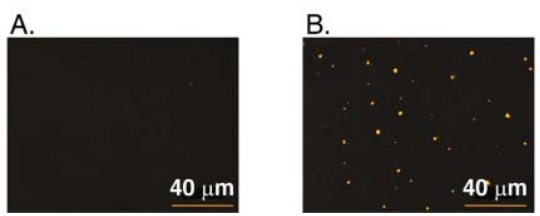

Fig. 1 (a) UV-Vis absorbance spectra, (b) TEM and (c) scattering images of solutions containing MPA-AuNPs in the (A) absence and (B) presence $(100 \mu \mathrm{M})$ of $\mathrm{Hg}^{2+}$. The concentration of the MPA-AuNPs was $3.0 \mathrm{nM}$. Inset a.: Photographic image of MPA-AuNP solutions in the (A) absence and (B) presence of $\mathrm{Hg}^{2+}(100 \mu \mathrm{M})$. Inset b.: Time-course measurement of $E x_{650 / 520}$ for MPA-AuNPs upon the addition of $\mathrm{Hg}^{2+}$ $(100 \mu \mathrm{M})$. Buffer: $50 \mathrm{mM}$ Tris-borate solution $(\mathrm{pH} 9.0)$. 
light scattered from single MPA-AuNPs was not readily observed by our DFS system. Note that strong scattering occurred once the MPA-AuNPs had aggregated; the orange and red spots correspond to scattering images of MPA-AuNP aggregates.

Fig. 2 compares the effect of the buffer composition on the sensing of four metal ions $\left(\mathrm{Sr}^{2+}, \mathrm{Pb}^{2+}, \mathrm{Hg}^{2+}\right.$ and $\left.\mathrm{Cd}^{2+}\right)$ using the MPA-AuNPs. For comparison, the as-prepared MPA-AuNPs in $50 \mathrm{mM}$ Tris-borate (in the absence of metal ions) was used as a control. Surprisingly, the buffer composition (Tris-borate, Tris$\mathrm{HCl}$ or borate- $\mathrm{NaOH} ; \mathrm{pH}$ 9.0) did play a significant role in affecting the sensing of these four metal ions; herein, we describe the concentrations of the Tris-borate and Tris- $\mathrm{HCl}$ buffers and the borate- $\mathrm{NaOH}$ buffer in terms of their molarities of Tris and borate, respectively. The MPA-AuNPs aggregated to varying degrees in solution through chelation in the presence of $\mathrm{Pb}^{2+}, \mathrm{Hg}^{2+}$ and $\mathrm{Cd}^{2+}$ and through counter ion pairing in the presence of $\mathrm{Sr}^{2+}$. As expected, in the buffers containing Tris (Tris-borate and Tris$\mathrm{HCl}$ ), the degree of aggregation of the as-prepared MPA-AuNPs with the four metal ions decreased in the following order: $\mathrm{Hg}^{2+}>$ $\mathrm{Cd}^{2+}>\mathrm{Pb}^{2+}>\mathrm{Sr}^{2+} \cdot{ }^{13}$ In the presence of borate, all four of these ions aggregated the MPA-AuNPs to a lesser degree; surprisingly, the sensing capability of the MPA-AuNPs for the four ions was lost almost completely in borate- $\mathrm{NaOH}$ solution. To understand the role that the buffer composition plays in determining the interactions of the MPA-AuNPs with the metal ions, we determined the zeta potentials of the as-modified MPA-AuNPs in $2 \mathrm{mM}$ sodium citrate solution (prior to preparation in the buffers) and in Tris-borate, Tris- $\mathrm{HCl}$ and borate- $\mathrm{NaOH}$ buffers; they were $-35,-23,-21$ and $-28 \mathrm{mV}$, respectively. The high zeta potential of the as-modified MPA-AuNPs indicates that high negative charge densities exist on their surfaces, mainly because of the adsorption of citrate $\left(\mathrm{p} K_{\mathrm{a} 3}=6.39\right)$ and MPA $\left(\mathrm{p} K_{\mathrm{a}}=4.87\right)$, which both dissociate under the experimental conditions. In the three buffer systems, the weakly bound citrate ions were displaced by such species as Tris $\left(\mathrm{p} K_{\mathrm{a}}=8.5\right)$ and chloride, hydroxide, borate $\left(\mathrm{p} K_{\mathrm{al}}=9.2\right)$ and sodium ions. We note that all of these bound species interact very weakly with the tested metal ions and MPA; thus, they do not have any dramatic impact on the interactions between MPA and the tested metal ions. Because the interactions of MPA with the metal ions in the three buffer systems were affected only slightly by changes in the ionic strength, we suggest that repulsion (charge screening) was the main reason why metal ion-induced aggregation (cross-linking of NPs) did not occur in the borate- $\mathrm{NaOH}$ solution. One other advantage of using Tris as the

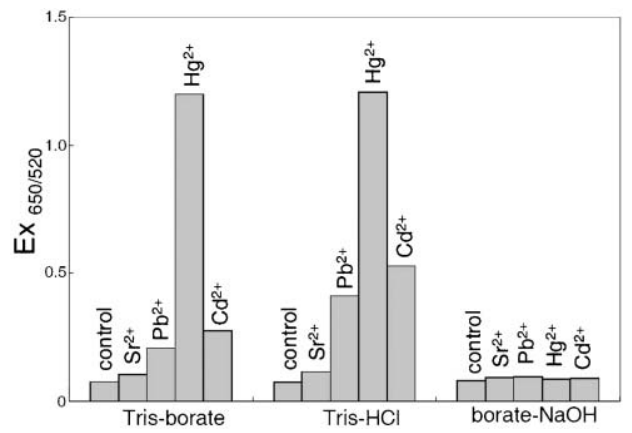

Fig. 2 Values of $E x_{650 / 520}$ ratios for the MPA-AuNPs in $50 \mathrm{mM}$ Trisborate, $50 \mathrm{mM}$ Tris- $\mathrm{HCl}$ and $10.1 \mathrm{mM}$ borate- $\mathrm{NaOH}$ after the addition of $100 \mu \mathrm{M}$ metal ions $\left(\mathrm{Sr}^{2+}, \mathrm{Pb}^{2+}, \mathrm{Hg}^{2+}\right.$ and $\left.\mathrm{Pb}^{2+}\right)$ at $\mathrm{pH}$ 9.0. buffer is its weak complexation with these metal ions; ${ }^{14}$ i.e., it does not interfere significantly with the MPA-AuNP/metal ion aggregation, but it prevents the formation of metal oxides and metal hydroxides that precipitate at high values of $\mathrm{pH}$. We note that precipitation did not occur in any of the three buffer systems. Fig. 2 also indicates that the interference from $\mathrm{Pb}^{2+}$ and $\mathrm{Cd}^{2+}$ in the determination of $\mathrm{Hg}^{2+}$ when using MPA-AuNPs in the Trisborate buffer was less than that in Tris- $\mathrm{HCl}$. This result suggests that it is possible to tune the metal ion sensing capability of the MPA-AuNPs simply by controlling the surface charge density through careful selection of a suitable buffer. In the following experiments, we used Tris-borate buffer to prepare all of the MPA-AuNP solutions.

Next, we recorded the UV-Vis spectra and zeta potentials of AuNP and MPA-AuNP solutions prepared in Tris-borate buffers of various concentrations in the absence and presence of $\mathrm{Hg}^{2+}$ ions $(5 \mu \mathrm{M})$. Increasing the Tris-borate concentration, leads to a greater amount of the weakly adsorbed citrate ions being replaced from the surfaces of the AuNPs and MPA-AuNPs by Tris and borate species. As a result, the zeta potentials of the AuNPs and MPAAuNPs decreased, as exhibited in Fig. 3, and eventually the NPs aggregated. The AuNPs and MPA-AuNPs aggregated in Trisborate solutions having concentrations above 25 and $100 \mathrm{mM}$, respectively; presumably, the MPA-AuNPs were more stable than the AuNPs at higher Tris-borate concentrations because of the ionized carboxylic acid units of their MPA moieties. Based on the stability of the MPA-AuNPs and the aggregation of the MPAAuNPs mediated by $\mathrm{Hg}^{2+}(5 \mu \mathrm{M})$, the optimized concentration of Tris-borate for our MPA-AuNP-based $\mathrm{Hg}^{2+}$ sensor was $50 \mathrm{mM}$.

Next, we investigated the optimum MPA density on the MPAAuNPs' surfaces for the binding of $\mathrm{Hg}^{2+}$ ions. Fig. S1 (ESI + ) displays the calibration curves of $\mathrm{Hg}^{2+}$ in various MPA-AuNP solutions. When using the solutions of MPA-AuNPs having $6.70 \times 10^{2}, 1.76 \times 10^{3}, 3.35 \times 10^{3}$ and $6.70 \times 10^{3}$ MPA ligands per NP, the $E x_{650 / 530}$ ratios reached plateaus at $\mathrm{Hg}^{2+}$ concentrations of $7.5,10,25$ and $50 \mu \mathrm{M}$, respectively. These results indicate that the MPA-AuNPs having lower MPA densities were more sensitive toward the $\mathrm{Hg}^{2+}$ ions. On the other hand, the MPAAuNPs having greater MPA densities had the ability to trap a larger number of $\mathrm{Hg}^{2+}$ ions, which is a useful feature for trapping purposes. A decrease in the number of receptor groups per NP reduces the redundancy in the metal ion based particle linking process and, thus, provides better sensitivity, ${ }^{8}$ but we found that

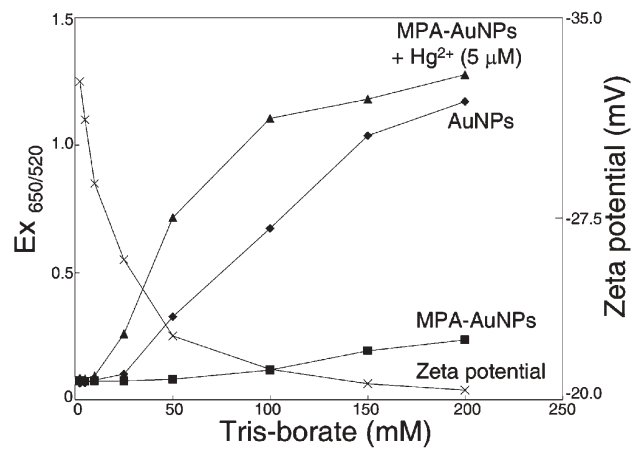

Fig. 3 Effect of the concentration of Tris-borate on the zeta potential of the MPA-AuNPs and on the $E x_{650 / 520}$ ratios of AuNPs and MPA-AuNPs (in the absence/presence of $5 \mu \mathrm{M} \mathrm{Hg}^{2+}$ ) at $\mathrm{pH}$ 9.0. 


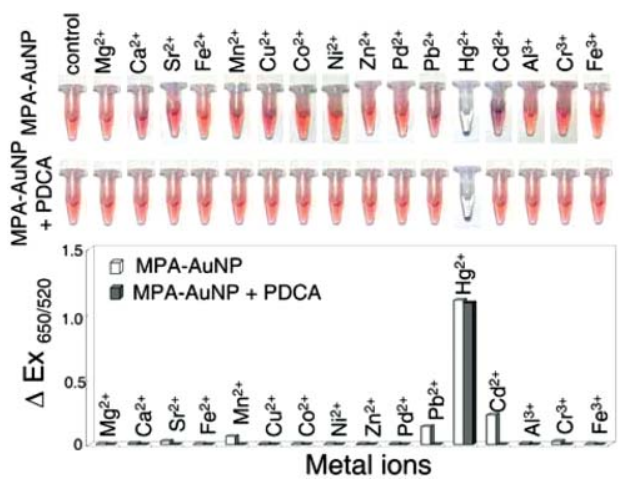

Fig. 4 (Top) Photographic images of the colors and (bottom) Ex $x_{650 / 520}$ differences of the MPA-AuNPs in the absence and presence of PDCA $(1.0 \mathrm{mM})$ after the addition of $100 \mu \mathrm{M}$ metal ions in $50 \mathrm{mM}$ Tris-borate solutions ( $\mathrm{pH}$ 9.0).

the MPA-AuNPs aggregated in $50 \mathrm{mM}$ Tris-borate ( $\mathrm{pH}$ 9.0) when the MPA density was less than $6.70 \times 10^{2}$ ligands per AuNP. Based on the stability of the MPA-AuNPs and their sensitivity toward the detection of $\mathrm{Hg}^{2+}$ ions in $50 \mathrm{mM}$ Tris-borate solution, the optimized ligand density was $6.70 \times 10^{2}$ MPA units per AuNP.

Under the optimum conditions, we tested the practicality of using the MPA-AuNPs for the sensing of various metal ions. Fig. 4 displays the changes in the colors and UV-vis absorption spectra of the MPA-AuNPs $(3.0 \mathrm{nM}) 2 \mathrm{~h}$ after adding various metal ions $(100 \mu \mathrm{M})$. In this series, we found that the presence of $\mathrm{Ca}^{2+}, \mathrm{Sr}^{2+}$, $\mathrm{Mn}^{2+}, \mathrm{Pb}^{2+}, \mathrm{Cd}^{2+}$ and $\mathrm{Cr}^{3+}$ ions led to increases in $E x_{650 / 520}$ to varying degrees, whereas the remaining ions exhibited no significant effects under identical conditions. These results suggest poor selectivity of the MPA-AuNP-based $\mathrm{Hg}^{2+}$ probe with respect to $\mathrm{Ca}^{2+}, \mathrm{Sr}^{2+}, \mathrm{Mn}^{2+}, \mathrm{Pb}^{2+}, \mathrm{Cd}^{2+}$ and $\mathrm{Cr}^{3+}$ ions. Fortunately, greater selectivity of the MPA-AuNP probe toward $\mathrm{Hg}^{2+}$ ions was readily achieved in the presence of the chelating ligand PDCA (1.0 mM). PDCA forms much more stable complexes with heavymetal ions, such as $\mathrm{Hg}^{2+}\left(\log \beta_{2}=20.28\right)$, than with other metal ions. ${ }^{15}$ To ensure better masking and the formation of stable complexes with $\mathrm{Hg}^{2+}$, we added PDCA to each MPA-AuNP solution at a concentration at least ca. 10 times greater than that $(100 \mu \mathrm{M})$ of $\mathrm{Hg}^{2+}$. As indicated in Fig. 4, MPA-AuNP in $50 \mathrm{mM}$ Tris-borate ( $\mathrm{pH}$ 9.0) containing $1.0 \mathrm{mM}$ PDCA responded selectively toward $\mathrm{Hg}^{2+}$ ions, by 100 -fold or more, relative to the other metal ions. We suggest that some PDCA ligands bound to the MPA-AuNP species through $\mathrm{Au}-\mathrm{N}$ bonds, ${ }^{16}$ improving the probes' selectivity toward $\mathrm{Hg}^{2+}$ ions through a cooperative effect, while the PDCA ligands in the bulk solutions formed complexes with the other metal ions, suppressing their interference with the probes. We performed a series of competitive experiments to test the practical applicability of our MPA-AuNP nanosensor for the selective colorimetric detection of $\mathrm{Hg}^{2+}$. After adding $\mathrm{Hg}^{2+}$ $(10 \mu \mathrm{M})$ and the interfering metal ions $\left(\mathrm{Ca}^{2+}, \mathrm{Sr}^{2+}, \mathrm{Mn}^{2+}, \mathrm{Pb}^{2+}\right.$, $\mathrm{Cd}^{2+}$ and $\left.\mathrm{Cr}^{3+} ; 100 \mu \mathrm{M}\right)$ to a mixture of MPA-AuNP and PDCA $(1.0 \mathrm{mM})$, we did not observe any significant interference in the detection of the $\mathrm{Hg}^{2+}$ ions. As indicated in Fig. 5, the $E x_{650 / 520}$ ratios of the MPA-AuNPs $(0.2 \mathrm{nM})$ increased upon increasing the concentration of $\mathrm{Hg}^{2+}$ ions. A linear correlation $\left(R^{2}=0.95\right)$ existed between the value of $E x_{650 / 520}$ and the concentration of $\mathrm{Hg}^{2+}$ ions

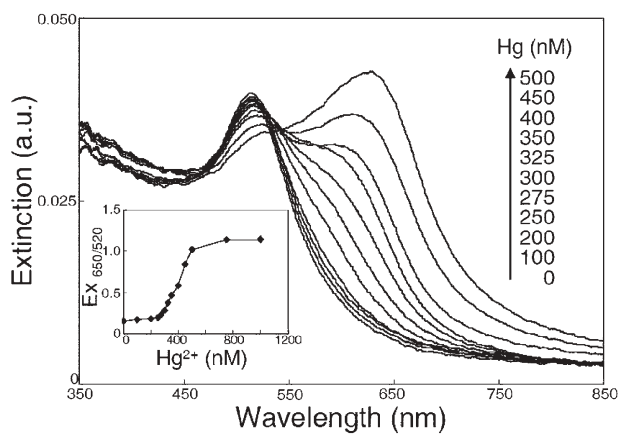

Fig. 5 UV-Vis absorption responses of MPA-AuNPs $(0.2 \mathrm{nM})$ after the addition of various concentrations of $\mathrm{Hg}^{2+}$ ions $(0,100,200,250,275,300$, $325,350,400,450$ and $500 \mathrm{nM})$. Inset: Plot of $E x_{650 / 520}$ of MPA-AuNPs as a function of the $\mathrm{Hg}^{2+}$ concentration.

over the range $250-500 \mathrm{nM}$. The LOD for $\mathrm{Hg}^{2+}$, at a signal-tonoise ratio of 3, was $100 \mathrm{nM}$. Thus, our approach pushes the sensitivity lower, by one order of magnitude, than those of other reported chelation/aggregation-mediated colorimetric AuNP-based sensors. ${ }^{8,12}$ Although the detected linear range of this MPAAuNP-based $\mathrm{Hg}^{2+}$ sensor was narrow, we believe that this range could be extended by modulating the MPA ligand density or the concentration of the Tris-borate buffer. More importantly, in the presence of PDCA, the MPA-AuNP nanosensor behaved almost completely free to interference from any other metal ion.

In conclusion, the high selectivity and sensitivity (LOD = $100 \mathrm{nM}$ ) of MPA-AuNPs for $\mathrm{Hg}^{2+}$ results from fine control of the buffer composite, the MPA ligand density on the MPA-AuNPs, and the excellent cooperativity of PDCA. We believe that this simple, low-cost approach may serve as a foundation for the preparation of practical nanosensors that will allow the rapid determination of $\mathrm{Hg}^{2+}$ concentrations in aqueous biological and environmental samples.

\section{Notes and references}

1 L. M. Campbell, D. G. Dixon and R. E. Hecky, J. Toxicol. Environ. Health B, 2003, 6, 325 .

2 Q. Wang, D. Kim, D. D. Dionysiou, G. A. Sorial and D. Timberlake, Environ. Pollut., 2004, 131, 323.

3 T. Morris and G. Szulczewski, Langmuir, 2002, 18, 5823.

4 E. M. Nolan, M. E. Racine and S. J. Lippard, Inorg. Chem., 2006, 45, 2742.

5 Y. Tang, F. He, M. Yu, F. Feng, L. An, H. Sun, S. Wang, Y. Li and D. Zhu, Macromol. Rapid Commun., 2006, 27, 389.

6 A. Ono and H. Togashi, Angew. Chem., Int. Ed., 2004, 43, 4300.

7 A. Moores and F. Goettmann, New J. Chem., 2006, 30, 1121.

8 Y. Kim, R. C. Johnson and J. T. Hupp, Nano Lett., 2001, 1, 165.

9 C. A. Mirkin, R. L. Letsinger, R. C. Mucic and J. J. Storhoff, Nature, 1996, 382, 607

10 H. Otsuka, Y. Akiyama, Y. Nagasaki and K. Kataoka, J. Am. Chem. Soc., 2001, 123, 8226

11 N. T. K. Thanh and Z. Rosenzweig, Anal. Chem., 2002, 74, 1624.

12 X. He, H. Liu, Y. Li, S. Wang, Y. Li, N. Wang, J. Xiao, X. Xu and D. Zhu, Adv. Mater., 2005, 17, 2811.

13 For acetate, the stability constants are: $\log K(\mathrm{~Pb})=4.1 ; \log K(\mathrm{Hg})=$ $10.1 ; \log K(\mathrm{Cd})=3.2 ;$ and $\log K(\mathrm{Sr})=1.1:$ F. M. M. Morel, in Principles of Aquatic Chemistry, Wiley-Interscience, New York, 1983, ch. 6, pp. 248-249.

14 P. J. Brignac, Jr. and C. Mo, Anal. Chem., 1975, 47, 1465.

15 E. Norkus, I. Stalnioniene and D. C. Crans, Heteroat. Chem., 2003, 14, 625.

16 R. D. Felice and A. Selloni, J. Chem. Phys., 2004, 120, 4906. 